\title{
Adaptive BLAST-type Decision-Feedback Equalizers for DS-CDMA Systems
}

\author{
Constantinos Rizogiannis*, Eleftherios Kofidis ${ }^{\dagger}$, Athanasios A. Rontogiannis ${ }^{\ddagger}$, and Sergios Theodoridis* \\ ${ }^{*}$ Dept. of Informatics and Telecommunications, \\ University of Athens, Panepistimioupolis, 15784 Athens, Greece. \\ Email: $\{$ krizog,stheodor $\} @$ di.uoa.gr \\ ${ }^{\dagger}$ Dept. of Statistics and Insurance Science, \\ University of Piraeus, 18534 Piraeus, Greece. \\ Email: kofidis@unipi.gr \\ ¥Institute for Space Applications and Remote Sensing, \\ National Observatory of Athens, 15236 P. Penteli, Greece. \\ E-mail: tronto@space.noa.gr
}

\begin{abstract}
In this paper, a new adaptive equalization scheme for time-varying and frequency selective channels in direct sequence code division multiple access (DS-CDMA) systems is proposed. It consists of a number of serially connected stages and detects users in an ordered manner, applying a decision feedback equalizer (DFE) at each stage. V-BLAST detection ordering is implemented, that is, the stronger signal is extracted first so that the weaker users can be more easily detected. Both the equalizer filters and the order in which the users are extracted are updated in a recursive least squares (RLS) manner, efficiently realized through time- and order-update recursions. Two variants of this equalization scheme are considered, with and without knowledge of the spreading sequences. Their bit error rate (BER) performance is evaluated via simulations, in a severe near-far environment, and their superiority over existing techniques is demonstrated.
\end{abstract}

\section{INTRODUCTION}

In a direct sequence-code division multiple access (DSCDMA) communication system, several users access the same channel using a common carrier frequency. A unique code sequence is assigned to each user, to spread the signal prior to transmission [1]. It is desirable that code sequences be orthogonal so that the users can be effectively separated at the receiver, eliminating inter-user interference. However, in realistic environments, asynchronous transmission and/or frequency selective channels result in time-shifted and scaled versions of users' code sequences, destroying orthogonality. Multiple access interference (MAI) and inter-symbol interference (ISI) are consequently generated. In a mobile environment, fading is also present and hence the receiver needs to have an adaptation capability as well.

It is well known [2] that when the users are received with different signal strengths, successive interference cancellation (SIC) is the preferrable solution, as it exhibits better near-far resistance. SIC consists of a cascade of stages, detecting one user at each stage. The stronger users are detected first and the detected signal is cancelled from the received signal so that the remaining users "see" less MAI. On the other hand, parallel interference cancellation (PIC) is preferred when all users have equal strength at the receiver [3]. An adaptive method performing successive interference cancellation in time-varying environments was proposed in [4], for additive white Gaussian noise (AWGN) and slow Rayleigh fading channels in a near-far situation, and shown to exhibit superior performance compared to the conventional SIC (CSIC) [2] receiver. An improved adaptive SIC (ASIC) technique was developed for AWGN as well as flat Rayleigh fading channels [5]. Its extension to frequency selective Rayleigh fading channels was presented in [6], where its superiority over the CSIC algorithm as well as a PIC method was demonstrated.

In this paper, a new adaptive equalization scheme of the SIC type is proposed for DS-CDMA systems operating over timevarying and frequency selective channels. Its development relies on the formulation of a DS-CDMA system as one with multiple inputs and multiple outputs (MIMO) and the adoption of existing adaptive solutions of the BLAST-type for MIMO channel equalization [7], [8]. The proposed equalizer consists of a number of serially connected stages and detects users in an ordered manner, applying a decision feedback equalizer (DFE) at each stage. V-BLAST [9] detection ordering is followed, that is, the stronger signal is extracted first, so that the weaker users can be more easily detected. Both the equalizer filters and the order in which the users are extracted are updated in a recursive least squares (RLS) manner, efficiently realized through time- and order-update recursions. Two variants of this equalization scheme are considered, with and without knowledge of the users' spreading sequences. Their BER performance is evaluated via simulations, in a severe nearfar environment, where their superiority, compared to ASIC and conventional techniques, is demonstrated.

Notation. $(\cdot)^{T}$ and $(\cdot)^{H}$ denote transpose and Hermitian transpose of a matrix, respectively. $\boldsymbol{I}_{m}$ is the $m$ th-order identity matrix, while $\mathbf{0}_{m \times n}$ denotes the $m \times n$ matrix of all zeros. Matlab notation is used to designate a submatrix of a given matrix. Complex conjugation is denoted by ${ }^{\star}$. $\otimes$ is the (left) Kronecker product. 


\section{SySTEM MODEL}

Consider the uplink of a symbol-synchronous DS-CDMA system with a spreading factor of $P$ chips per symbol, $K$ single-antenna users, and a single-antenna receiver. The users transmit independently the symbol sequences $\boldsymbol{s}_{i}(k), i=1,2, \ldots, K$. Each such sequence is spread through a $P$-periodic spreading code $\boldsymbol{c}_{i}=$ $\left[\begin{array}{llll}c_{i}(0) & c_{i}(1) & \cdots & c_{i}(P-1)\end{array}\right]^{T}$. The transmission is through frequency selective channels, with impulse responses $\boldsymbol{h}_{i}(k)=\left[\begin{array}{llll}h_{i, 0}(k) & h_{i, 1}(k) & \cdots & h_{i, L-1}(k)\end{array}\right]^{T}$, $i=1,2, \ldots, K$, of length $L$, that are assumed to vary at the symbol rate.

The discrete-time signal $x$ at the receiver's front-end results from the summation of signals from all $K$ users, corrupted by additive white Gaussian noise (AWGN). Sampling at chip rate and collecting $P$ successive measurements of $x$ in a $P \times 1$ vector, a multiple-input multiple-output (MIMO) formulation with $K$ inputs and $P$ outputs results for the DS-CDMA system. With the channels being constant during each symbol interval, the following input-output relationship results:

$$
\begin{aligned}
\boldsymbol{x}(k) & =\left[\begin{array}{llll}
x(k P) & x(k P+1) & \cdots & x(k P+P-1)
\end{array}\right]^{T} \\
& =\sum_{i=1}^{K} A_{i}\left[s_{i}(k-1) \mathcal{C}_{i}^{\mathrm{p}}+s_{i}(k) \mathcal{C}_{i}^{\mathrm{c}}\right] \boldsymbol{h}_{i}(k)+\boldsymbol{v}(k),(1)
\end{aligned}
$$

where $A_{i}$ is the amplitude of the $i$ th user's signal, $s_{i}(k-1)$ and $s_{i}(k)$ are the input symbols of the $i$ th user at time instances $k-1$ and $k$, respectively, $\boldsymbol{v}(k)$ is additive zero mean, white, complex Gaussian noise vector, and the $P \times L$ matrices $\mathcal{C}_{i}^{\mathrm{p}}, \mathcal{C}_{i}^{\mathrm{c}}$ are built from the $(P+L-1) \times L$ convolution matrix

$$
\mathcal{C}_{i}=\left[\begin{array}{ccc}
c_{i}(0) & & \mathbf{0}_{(L-1) \times 1} \\
\vdots & \ddots & c_{i}(0) \\
c_{i}(P-1) & & \vdots \\
\mathbf{0}_{(L-1) \times 1} & \ddots & c_{i}(P-1)
\end{array}\right]
$$

as

$$
\begin{aligned}
\mathcal{C}_{i}^{\mathrm{p}} & =\left[\begin{array}{c}
\mathcal{C}_{i}(P+1: P+L-1,:) \\
\mathbf{0}_{(P-L+1) \times L}
\end{array}\right], \\
\mathcal{C}_{i}^{\mathrm{c}} & =\mathcal{C}_{i}(1: P,:)
\end{aligned}
$$

Similarly with equation (1), but collecting $P+L-1$ successive samples of $x$ instead of $P$, a new MIMO formulation with $K$ inputs and $P+L-1$ outputs results, as follows

$$
\begin{aligned}
& \overline{\boldsymbol{x}}(k)= \\
& {\left[\begin{array}{llll}
x(k P) & x(k P+1) & \cdots & x(k P+P-1)
\end{array}\right.} \\
& x((k+1) P) \quad \cdots \quad x((k+1) P+L-2)]^{T} \\
& =\sum_{i=1}^{K} A_{i}\left[s_{i}(k-1) \overline{\mathcal{C}}_{i}^{\mathrm{p}} \boldsymbol{h}_{i}(k)+s_{i}(k) \overline{\mathcal{C}}_{i}^{\mathrm{c}} \boldsymbol{h}_{i}(k)+\right. \\
& \left.s_{i}(k) \overline{\mathcal{C}}_{i}^{\mathrm{cn}} \boldsymbol{h}_{i}(k+1)+s_{i}(k+1) \overline{\mathcal{C}}_{i}^{\mathrm{n}} \boldsymbol{h}_{i}(k+1)\right]+\overline{\boldsymbol{v}}(k),
\end{aligned}
$$

where $\overline{\mathcal{C}}_{i}^{\mathrm{p}}=\left[\begin{array}{cc}\mathcal{C}_{i}^{T}(P+1: P+L-1,:) & \mathbf{0}_{P \times L}^{T}\end{array}\right]^{T}, \overline{\mathcal{C}}_{i}^{\mathrm{c}}=$

$$
\begin{aligned}
& {\left[\begin{array}{ll}
\mathcal{C}_{i}^{T}(1: P,:) & \mathbf{0}_{(L-1) \times L}^{T}
\end{array}\right]^{T} \text {, and }} \\
& \overline{\mathcal{C}}_{i}^{\mathrm{cn}}=\left[\begin{array}{c}
\mathbf{0}_{P \times L} \\
\mathcal{C}_{i}(P+1: P+L-1,:)
\end{array}\right], \\
& \overline{\mathcal{C}}_{i}^{\mathrm{n}}=\left[\begin{array}{c}
\mathbf{0}_{P \times L} \\
\mathcal{C}_{i}(1: L-1,:)
\end{array}\right] .
\end{aligned}
$$

$\overline{\boldsymbol{v}}(k)$ is defined similarly with $\boldsymbol{v}(k)$. Note that the last $L-1$ elements of $\overline{\boldsymbol{x}}(k)$ equal the first $L-1$ elements of $\overline{\boldsymbol{x}}(k+1)$. The matrices $\overline{\mathcal{C}}_{i}^{\mathrm{p}}, \overline{\mathcal{C}}_{i}^{\mathrm{n}}$ are associated with the contributions from the preceding $\left(s_{i}(k-1)\right)$ and following $\left(s_{i}(k+1)\right)$ symbols, respectively. The current symbol contribution is associated with the matrices $\overline{\mathcal{C}}_{i}^{\mathrm{c}}$ and $\overline{\mathcal{C}}_{i}^{\mathrm{cn}}$. Observe that, in a slowly fading scenario, i.e., when $\boldsymbol{h}_{i}(k+1) \approx \boldsymbol{h}_{i}(k)$, the corresponding term becomes $s_{i}(k)\left(\overline{\mathcal{C}}_{i}^{\mathrm{c}}+\overline{\mathcal{C}}_{i}^{\mathrm{cn}}\right) \boldsymbol{h}_{i}(k)$, where it should be noted that $\overline{\mathcal{C}}_{i}^{\mathrm{c}}+\overline{\mathcal{C}}_{i}^{\mathrm{cn}}=\mathcal{C}_{i}$. This is a key observation for developing the equalization algorithm in the next section.

\section{BLAST-TYPE DFE SCHEME FOR TIME-VARYING CHANNELS}

Based on the equivalence between V-BLAST [9] and generalized DFE (GDFE) [10], an adaptive MIMO DFE detection scheme with variable detection order was proposed in [7] for flat time-varying channels. An extension of this method to include frequency selective channels was developed in [8], where expanded input and weight vectors are used in order to eliminate both MAI and ISI. This technique was shown to perform similarly to the V-BLAST algorithm with RLS channel tracking but at a significantly reduced computational complexity.

Making use of the MIMO formulation presented in the previous section, a similar equalization scheme can result for a DS-CDMA system with $K$ users and spreading factor $P$. The MIMO formulation of (5) will be first used here, where a DS-CDMA system is represented as a MIMO system with $K$ inputs and $P+L-1$ outputs. All code sequences will be assumed to be known at the receiver. The structure of the proposed adaptive scheme is shown in Fig. 1. The receiver detects the users in an ordered manner through $K$ successive stages. The stronger users, which are affected less by MAI, are detected first, allowing easier detection for the weaker users. At each stage, only one user is detected and the remaining users comprise the set of candidate users for the next stage. A DFE is applied at each stage in order to remove MAI and ISI. Let $\left\{o_{1}(k), \ldots, o_{K}(k)\right\}, o_{i}(k) \in$ $\{1, \ldots, K\}$ be the order in which the users are detected at time $k$. Let us denote the set of candidate users for stage $i$ by $U_{i}(k)=\{1,2, \ldots, K\}-\left\{o_{1}(k), o_{2}(k), \ldots, o_{i-1}(k)\right\}$. Although the ordering of users depends on time $k$, we will skip this notation for the sake of simplicity. Thus, henceforth, $o_{i}$ will denote the user assigned to the $i$ th stage at time $k$, unless otherwise stated.

Note from Fig. 1 that the input signal to the feedforward filter is modified according to the RAKE receiver idea [1]. Given that the $o_{i}$ th user is to be detected at stage $i$, the input 


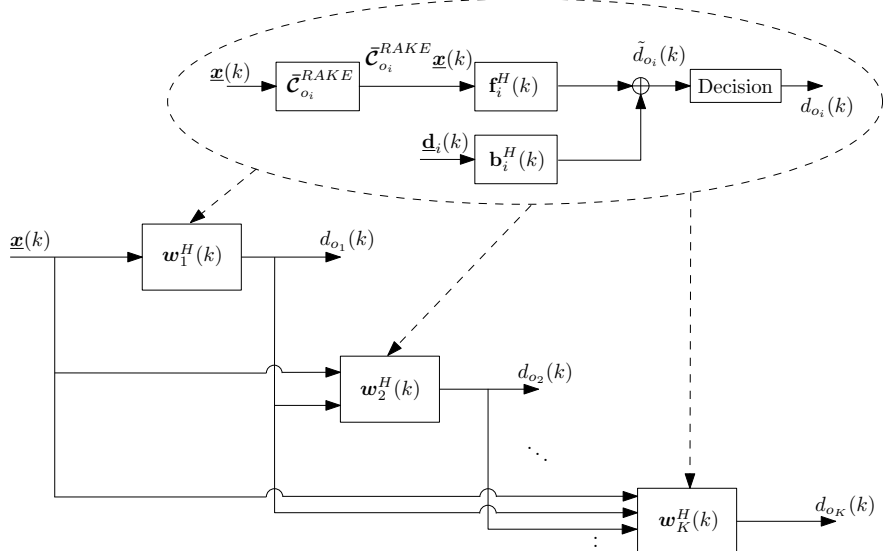

Fig. 1. Structure of the new adaptive DFE equalizer incorporating the knowledge of the code sequences.

of the feedforward filter consists of the last $K_{\mathrm{f}}$ received vectors $\overline{\boldsymbol{x}}$, transformed as $\overline{\mathcal{C}}_{O_{i}}^{\mathrm{RAKE}} \underline{\boldsymbol{x}}(k)$, where

$\underline{\boldsymbol{x}}(k)=\left[\begin{array}{llll}\overline{\boldsymbol{x}}^{T}\left(k-K_{\mathrm{f}}+1\right) & \overline{\boldsymbol{x}}^{T}\left(k-K_{\mathrm{f}}+2\right) & \cdots & \overline{\boldsymbol{x}}^{T}(k)\end{array}\right]^{T}$

$\overline{\boldsymbol{x}}(k)$ is given by (5), and $\overline{\mathcal{C}}_{o_{i}}^{\mathrm{RAKE}}$ is the $K_{\mathrm{f}} L \times K_{\mathrm{f}}(P+L-1)$ block Toeplitz matrix

$$
\overline{\mathcal{C}}_{o_{i}}^{\mathrm{RAKE}}=\boldsymbol{I}_{K_{\mathrm{f}}} \otimes \mathcal{C}_{o_{i}}^{\mathrm{RAKE}}
$$

with $\mathcal{C}_{O_{i}}^{\mathrm{RAKE}}=\mathcal{C}_{o_{i}}^{T}$. This way, advantage is taken of the known code sequences to lessen the effect of the other users. The equalizer filter $\boldsymbol{w}_{i}(k)$ of the $i$ th stage DFE is composed of the $K_{\mathrm{f}} L$-dimensional feedforward filter $\boldsymbol{f}_{i}(k)$ and the $\left(K K_{\mathrm{b}}+\right.$ $i-1)$-dimensional feedback filter $\boldsymbol{b}_{i}(k)$, as in

$$
\boldsymbol{w}_{i}(k)=\left[\begin{array}{ll}
\boldsymbol{f}_{i}^{T}(k) & \boldsymbol{b}_{i}^{T}(k)
\end{array}\right]^{T}, i=1,2, \ldots, K .
$$

The input of the feedback filter at stage $i$ is the $\left(K K_{\mathrm{b}}+i-1\right) \times$ 1 vector $\underline{\boldsymbol{d}}_{i}(k)$ which contains the decisions for all users from $K_{\mathrm{b}}$ previous time instances and the current data decisions from the $i-1$ previous stages. If the output of the $i$ th stage equalizer, assigned to user $o_{i}$, is $\tilde{d}_{o_{i}}(k)$ and $d_{o_{i}}(k)=f\left[\tilde{d}_{o_{i}}(k)\right]$ is the corresponding decision device output, then the input of the feedback filter is written as

$$
\begin{aligned}
& \underline{\boldsymbol{d}}_{i}(k)=\left[\begin{array}{lll}
\boldsymbol{d}^{T}\left(k-K_{\mathrm{b}}\right) & \cdots & \boldsymbol{d}^{T}(k-1)
\end{array}\right. \\
& \left.d_{o_{1}}(k) \quad d_{o_{2}}(k) \quad \cdots \quad d_{o_{i-1}}(k)\right]^{T}(11)
\end{aligned}
$$

where

$$
\boldsymbol{d}(k)=\left[\begin{array}{llll}
d_{1}(k) & d_{2}(k) & \cdots & d_{K}(k)
\end{array}\right]^{T}
$$

contains the decisions for all $K$ users at time $k$.

Using the above definitions, the output of the $i$ th DFE can be written as

$$
\begin{aligned}
\tilde{d}_{o_{i}}(k) & =\boldsymbol{f}_{i}^{H}(k) \overline{\boldsymbol{\mathcal { C }}}_{o_{i}}^{\mathrm{RAKE}} \underline{\boldsymbol{x}}(k)+\boldsymbol{b}_{i}^{H}(k) \underline{\boldsymbol{d}}_{i}(k) \\
& =\boldsymbol{w}_{i}^{H}(k) \boldsymbol{C}_{i, o_{i}} \boldsymbol{y}_{i}(k),
\end{aligned}
$$

where

$$
\boldsymbol{C}_{i, o_{i}}=\left[\begin{array}{cc}
\overline{\mathcal{C}}_{o_{i}}^{\mathrm{RAKE}} & \mathbf{0} \\
\mathbf{0} & \boldsymbol{I}_{K K_{\mathrm{b}}+i-1}
\end{array}\right]
$$

and

$$
\boldsymbol{y}_{i}(k)=\left[\begin{array}{ll}
\underline{\boldsymbol{x}}^{T}(k) & \underline{\boldsymbol{d}}_{i}^{T}(k)
\end{array}\right]^{T}, \quad i=1,2, \ldots, K
$$

is the $(V+i-1) \times 1$ input vector of the $i$ th stage DFE, where $V=K_{\mathrm{f}}(P+L-1)+K K_{\mathrm{b}}$.

In a time-varying environment, the equalizer filters as well as the detection order need to be updated in time. In the proposed scheme, the minimization of a LS cost function is used to meet both requirements. To compute the equalizer of the $i$ th stage, we have to update all equalizers corresponding to the set of candidate users $U_{i}(k)$ in order to determine which user should be extracted (in a BLAST-like fashion). The equalizer $\boldsymbol{w}_{i, j}^{H}(k)$, corresponding to the $i$ th stage and the $j$ th user, is the one minimizing the following cost function

$$
\mathcal{E}_{i, j}(k)=\sum_{l=1}^{k} \lambda^{k-l}\left|d_{j}(l)-\boldsymbol{w}_{i, j}^{H}(k) \boldsymbol{C}_{i, j} \boldsymbol{y}_{i}(l)\right|^{2},
$$

where $0<\lambda \leq 1$ is the forgetting factor and the matrix $C_{i, j}$ has the structure of $C_{i, o_{i}}$ in (14) but, for the $i$ th stage and the $T$ th user, it is given by

$$
\boldsymbol{C}_{i, j}=\left[\begin{array}{cc}
\overline{\mathcal{C}}_{j}^{\mathrm{RAKE}} & \mathbf{0} \\
\mathbf{0} & \boldsymbol{I}_{K K_{\mathrm{b}}+i-1}
\end{array}\right]=\left[\begin{array}{cc}
\boldsymbol{C}_{i-1, j} & \mathbf{0} \\
\mathbf{0} & 1
\end{array}\right]
$$

After having updated all tentative equalizers, $\boldsymbol{w}_{i, j}(k)$ for $j \in U_{i}(k)$, the one achieving the lowest squared error is finally applied at the current stage. In other words, we set

$$
\begin{aligned}
o_{i} & =\arg \min _{j \in U_{i}(k)} \mathcal{E}_{i, j}(k), \\
\boldsymbol{w}_{i}(k) & =\boldsymbol{w}_{i, o_{i}}(k), \\
\mathcal{E}_{i}(k) & =\mathcal{E}_{i, o_{i}}(k) .
\end{aligned}
$$

The minimization of (16) with respect to $\boldsymbol{w}_{i, j}(k)$ is known [11] to result in

$$
\boldsymbol{w}_{i, j}(k)=\boldsymbol{\Phi}_{i, j}^{-1}(k) \boldsymbol{C}_{i, j} \boldsymbol{z}_{i, j}(k),
$$

where

$$
\boldsymbol{\Phi}_{i, j}(k)=\boldsymbol{C}_{i, j} \boldsymbol{\Phi}_{i}(k) \boldsymbol{C}_{i, j}^{H},
$$

and

$$
\begin{aligned}
& \boldsymbol{\Phi}_{i}(k)=\sum_{l=1}^{k} \lambda^{k-l} \boldsymbol{y}_{i}(l) \boldsymbol{y}_{i}^{H}(l), \\
& \boldsymbol{z}_{i, j}(k)=\sum_{l=1}^{k} \lambda^{k-l} \boldsymbol{y}_{i}(l) d_{j}^{*}(l) .
\end{aligned}
$$

As it can be seen from (19) and (22), to compute the tentative equalizers $\boldsymbol{w}_{i, j}$ at stage $i$, current decisions from all users must be known. In the training mode, these are provided by the training sequence. To overcome this causality problem in the decision-directed mode, we assume, as in [7], that the decisions at time $k$ are extracted using the optimum equalizers and detection ordering found at time $k-1$.

From (15) one can easily see that the equalizer input vector can be expressed in the following order-recursive manner:

$$
\boldsymbol{y}_{i+1}(k)=\left[\begin{array}{ll}
\boldsymbol{y}_{i}^{T}(k) & d_{o_{i}}(k)
\end{array}\right]^{T}
$$


Using this relation in the above, one can arrive at

$$
\boldsymbol{\Phi}_{i+1, j}(k)=\left[\begin{array}{cc}
\boldsymbol{\Phi}_{i, j}(k) & \boldsymbol{C}_{i, j} \boldsymbol{z}_{i, o_{i}}(k) \\
\boldsymbol{z}_{i, o_{i}}^{H}(k) \boldsymbol{C}_{i, j}^{H} & \alpha_{o_{i}}(k)
\end{array}\right],
$$

where

$$
\alpha_{j}(k)=\sum_{l=1}^{k} \lambda^{k-l}\left|d_{j}(l)\right|^{2} .
$$

Invoking the matrix inversion lemma [11], the inverse of $\boldsymbol{\Phi}_{i+1, j}(k)$ can be written as (26) (see next page) where

$$
\boldsymbol{\Gamma}_{i, j}(k)=\boldsymbol{\Phi}_{i, j}^{-1}(k) \boldsymbol{C}_{i, j}
$$

and

$$
\beta_{i, j}(k)=\frac{1}{\alpha_{o_{i}}(k)-\boldsymbol{z}_{i, o_{i}}^{H}(k) \boldsymbol{C}_{i, j}^{H} \boldsymbol{\Gamma}_{i, j}(k) \boldsymbol{z}_{i, o_{i}}(k)} .
$$

$\boldsymbol{\Gamma}_{i, j}(k)$ can be order-updated. Indeed, using (17), (26) in (27), we get (29) (see next page). Moreover, an analytical calculation of $\boldsymbol{\Gamma}_{1, j}(k)$ is necessary at every time instant. By defining the $(V+K) \times K$ matrix (30) (see next page) one can easily find from (22) and (30) that

$$
\begin{aligned}
& \boldsymbol{z}_{i, j}(k)=\left[\begin{array}{lll}
q_{1, j}(k) & \cdots & q_{V, j}(k)
\end{array}\right. \\
& \left.q_{V+o_{1}, j}(k) \quad \cdots \quad q_{V+o_{i-1}, j}(k)\right]^{T}(31)
\end{aligned}
$$

and

$$
\boldsymbol{z}_{i+1, j}(k)=\left[\begin{array}{cc}
\boldsymbol{z}_{i, j}^{T}(k) & q_{V+o_{i}, j}(k)
\end{array}\right]^{T},
$$

where $q_{m, n}(k)$ is the $(m, n)$ entry of $\boldsymbol{Q}(k)$. Moreover, it is straightforward to see that $\alpha_{j}(k)=q_{V+j, j}(k)$. Finally, an efficient order-update relation for the equalizer weights can be obtained by substituting (29) and (32) in (19):

$$
\begin{aligned}
& \boldsymbol{w}_{i+1, j}(k) \\
& =\left[\begin{array}{c}
\boldsymbol{w}_{i, j}(k) \\
0
\end{array}\right]+\beta_{i, j}(k) \Delta_{i, j}(k)\left[\begin{array}{c}
-\boldsymbol{\Gamma}_{i, j}(k) \boldsymbol{z}_{i, o_{i}}(k) \\
1
\end{array}\right]
\end{aligned}
$$

where

$$
\Delta_{i, j}(k)=q_{V+o_{i}, j}(k)-\boldsymbol{z}_{i, o_{i}}^{H}(k) \boldsymbol{C}_{i, j}^{H} \boldsymbol{\Gamma}_{i, j}(k) \boldsymbol{z}_{i, j}(k) .
$$

In order to determine the optimum detection ordering, the LS error energies must be computed. From (16), (25) and (30), and after some algebra, one can readily arrive at

$$
\mathcal{E}_{i, j}(k)=q_{V+j, j}(k)-\boldsymbol{w}_{i, j}^{H}(k) \boldsymbol{C}_{i, j} \boldsymbol{z}_{i, j}(k) .
$$

An efficient order-recursive formula for the LS error energies $\mathcal{E}_{i+1, j}(k)$ can also be derived:

$$
\mathcal{E}_{i+1, j}(k)=\mathcal{E}_{i, j}(k)-\beta_{i, j}(k) \Delta_{i, j}^{2}(k) .
$$

The order of detection is determined if we sort energies in increasing order. The user attaining the minimum energy is detected at the next stage.

At every time instant, knowledge of the first-stage filters $\boldsymbol{w}_{1, j}(k), j=1,2, \ldots, K$, is also necessary. This can be acquired through time-updating $\boldsymbol{w}_{1, j}(k)$ using the RLS recursion [11]:

$$
\begin{aligned}
\boldsymbol{p}_{j}(k) & =\boldsymbol{\Phi}_{1, j}^{-1}(k-1) \boldsymbol{C}_{1, j} \boldsymbol{y}_{1}(k), \\
\boldsymbol{K}_{j}(k) & =\frac{\lambda^{-1} \boldsymbol{p}_{j}(k)}{1+\lambda^{-1} \boldsymbol{y}_{1}^{H}(k) \boldsymbol{C}_{1, j}^{H} \boldsymbol{p}_{j}(k)} \\
\boldsymbol{\Phi}_{1, j}^{-1}(k) & =\lambda^{-1} \boldsymbol{\Phi}_{1, j}^{-1}(k-1)-\lambda^{-1} \boldsymbol{K}_{j}(k) \boldsymbol{p}_{j}^{H}(k), \\
\boldsymbol{w}_{1, j}(k) & =\boldsymbol{w}_{1, j}(k-1)+\boldsymbol{K}_{j}(k) e_{j}^{*}(k),
\end{aligned}
$$

where

$$
e_{j}(k)=d_{j}(k)-\boldsymbol{w}_{1, j}^{H}(k-1) \boldsymbol{C}_{1, j} \boldsymbol{y}_{1}(k)
$$

is the a priori estimation error. The above algorithm will henceforth be referred to as RAKE-RLS.

Alternatively, adopting the MIMO formulation of (1) and replacing in (9) $\mathcal{C}_{O_{i}}^{\mathrm{RAKE}}$ by $\left[\begin{array}{ll}\boldsymbol{I}_{P} & \mathbf{0}_{P \times(L-1)}\end{array}\right]$, a variant of the above scheme results, which does not explicitly utilize the spreading codes. A further improved version, with lower complexity and increased numerical robustness, is then easily derived by following the steps of [8], where the method of [7] was revisited for frequency selective channels, based on the updating of the inverse Cholesky factor of the input autocorrelation matrix. The resulting algorithm will be called square root multiuser detection (SR-MUD). It is important to emphasize that it does not require the spreading sequences to be known at the receiver.

\section{Simulations}

In this section, the performance of the proposed equalization algorithms is evaluated via computer simulations, in terms of bit error rate (BER). Each user generates QPSK symbols of duration $T_{s}=0.2 \mu \mathrm{sec}$, which are then spread using normalized Walsh-Hadamard code sequences. The generated sequences are transmitted over independent, time-varying and frequency selective channels with Rayleigh-faded paths of an exponential profile. A carrier frequency of $2.4 \mathrm{GHz}$ and a mobile speed of $50 \mathrm{~km} / \mathrm{h}$ is assumed, yielding a normalized Doppler frequency of about $f_{D} T_{s}=2.2 \times 10^{-5}$. For both RAKE-RLS and SR-MUD, a forgetting factor of 0.998 was seen to lead to good performance under these conditions, while the feedforward and feedback filters have a temporal span of $K_{\mathrm{f}}=1$ and $K_{\mathrm{b}}=1$ taps, respectively. ${ }^{1}$

The performance of the proposed schemes is compared with the RAKE receiver, the ASIC algorithm, and the linear receiver adapted via the exponentially weighted conventional RLS. The single user bound (SUB), representing the performance of a single-user system in a non-fading AWGN channel, is also shown as a benchmark. For the RAKE receiver, an LMS algorithm [11] is employed to track the channel variations with a step-size parameter of $5 \times 10^{-3}$ while for the ASIC

\footnotetext{
${ }^{1}$ This is quite reasonable, since, for $L \leq P$, at most one previous symbol appears at each received vector in (1) and (5).
} 


$$
\begin{aligned}
& \boldsymbol{\Phi}_{i+1, j}^{-1}(k)=\left[\begin{array}{cc}
\boldsymbol{\Phi}_{i, j}^{-1}(k)+\beta_{i, j}(k) \boldsymbol{\Gamma}_{i, j}(k) \boldsymbol{z}_{i, o_{i}}(k) \boldsymbol{z}_{i, o_{i}}^{H}(k) \boldsymbol{\Gamma}_{i, j}^{H}(k) & -\beta_{i, j}(k) \boldsymbol{\Gamma}_{i, j}(k) \boldsymbol{z}_{i, o_{i}}(k) \\
-\beta_{i, j}(k) \boldsymbol{z}_{i, o_{i}}^{H}(k) \boldsymbol{\Gamma}_{i, j}^{H}(k) & \beta_{i, j}(k)
\end{array}\right] \\
& \boldsymbol{\Gamma}_{i+1, j}(k)=\left[\begin{array}{cc}
\boldsymbol{\Gamma}_{i, j}(k)+\beta_{i, j}(k) \boldsymbol{\Gamma}_{i, j}(k) \boldsymbol{z}_{i, o_{i}}(k) \boldsymbol{z}_{i, o_{i}}^{H}(k) \boldsymbol{C}_{i, j}^{H} \boldsymbol{\Gamma}_{i, j}(k) & -\beta_{i, j}(k) \boldsymbol{\Gamma}_{i, j}(k) \boldsymbol{z}_{i, o_{i}}(k) \\
-\beta_{i, j}(k) \boldsymbol{z}_{i, o_{i}}^{H}(k) \boldsymbol{C}_{i, j}^{H} \boldsymbol{\Gamma}_{i, j}(k) & \beta_{i, j}(k)
\end{array}\right] \\
& \boldsymbol{Q}(k)=\sum_{l=1}^{k} \lambda^{k-l}\left[\begin{array}{lllll}
\underline{\boldsymbol{x}}^{T}(l) & \boldsymbol{d}^{T}\left(l-K_{\mathrm{b}}\right) & \cdots & \boldsymbol{d}^{T}(l-1) & \boldsymbol{d}^{T}(l)
\end{array}\right]^{T} \boldsymbol{d}^{H}(l) \\
& =\lambda \boldsymbol{Q}(k-1)+\left[\begin{array}{lllll}
\underline{\boldsymbol{x}}^{T}(k) & \boldsymbol{d}^{T}\left(k-K_{\mathrm{b}}\right) & \cdots & \boldsymbol{d}^{T}(k-1) & \boldsymbol{d}^{T}(k)
\end{array}\right]^{T} \boldsymbol{d}^{H}(k)
\end{aligned}
$$

it is set to $5 \times 10^{-4}$. In our experiments, we simulate a nearfar scenario, where the received amplitude of each user is determined such that $10 \log _{10}\left(A_{i} / A_{i+1}\right)^{2}=N \mathrm{~dB}$, and the amplitude of the first user is set to 1 . In all simulations, 200 frames of 2048 symbols each, with the first 256 devoted to training, were transmitted to obtain the results.

The BER performance versus $E_{b} / N_{0}(\mathrm{~dB})$ is depicted in Fig. 2 for $K=7, L=6, N=2 \mathrm{~dB}$, and for two different values of spreading factor $P$. As one can see, the superiority of the proposed schemes is evident in the higher $E_{b} / N_{0}$ regime. Specifically, for small values of $P$ (e.g., $P=8$ ) and at high $E_{b} / N_{0}$, SR-MUD outperforms RAKERLS, while both of them achieve better performance compared to the other algorithms. Moreover, for large values of $P$ (e.g., $P=128)$, RAKE-RLS attains the best performance, while the performance of SR-MUD and conventional RLS worsens significantly. The RAKE receiver and ASIC, although they perform better for larger spreading factors, they are always inferior to RAKE-RLS and SR-MUD in the higher $E_{b} / N_{0}$ regime.

The above results suggest that RAKE-RLS can operate efficiently under near-far conditions and in a time-varying, frequency selective environment, especially in the medium to high $E_{b} / N_{0}$ region and for larger spreading factors. SR-MUD, which needs no knowledge of the spreading codes, seems to perform better for smaller values of the spreading factor and at high $E_{b} / N_{0}(>20 \mathrm{~dB})$.

\section{REFERENCES}

[1] S. Verdú, Multiuser Detection, Cambridge University Press, 1998.

[2] P. Patel and J. Holtzman, "Analysis of a simple successive interference cancellation scheme in a DS-CDMA system," IEEE Journal on Selected Areas in Communications, June 1994.

[3] Y. D. Li and T. T. Tjhung, "Performance analysis of PIC-CDMA systems," Proc. ICCS-2002.

[4] Y. Cho and J. H. Lee, "Analysis of an adaptive SIC for near-far resistant DS-CDMA,” IEEE Trans. Communications, Nov. 1998.

[5] K.-C. Lai and J. J. Shynk, "Steady-state analysis of the adaptive successive interference canceler for DS/CDMA signals," IEEE Trans. Signal Processing, Oct. 2001.

[6] K.-C. Lai et al., "Adaptive successive interference cancellation for the IS-95 uplink," Proc. VTC-2000 (Fall).

[7] J. Choi, H. Yu, and Y. H. Lee, "Adaptive MIMO decision feedback equalization for receivers with time-varying channels," IEEE Trans. Signal Processing, Nov. 2005.

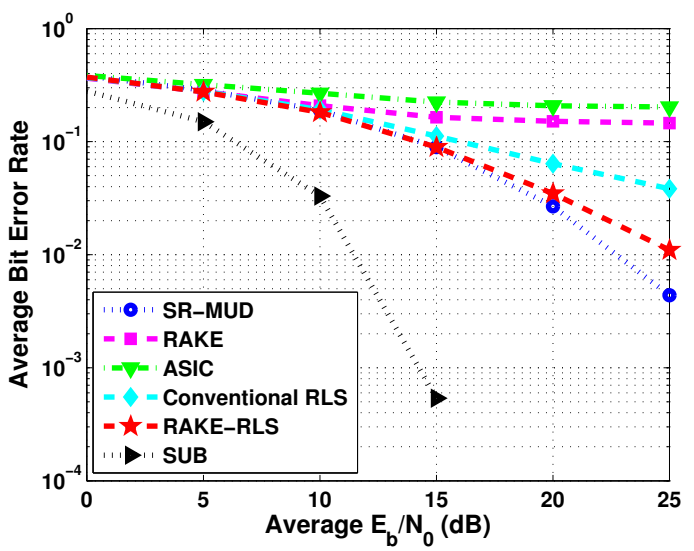

(a)

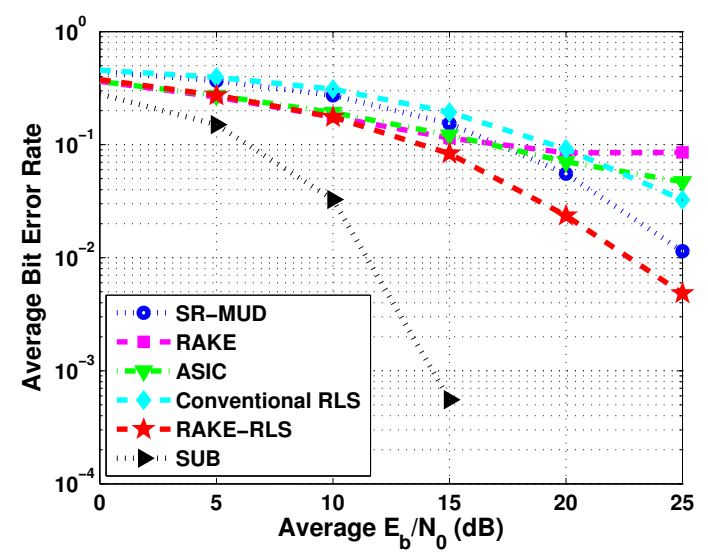

(b)

Fig. 2. BER vs. $E_{b} / N_{0}(\mathrm{~dB})$ for $K=7, L=6, N=2 \mathrm{~dB}$ and spreading factor (a) $P=8$, (b) $P=128$.

[8] V. Kekatos, A. A. Rontogiannis, and K. Berberidis, "Cholesky factorization-based adaptive BLAST DFE for wideband MIMO channels," EURASIP Journal on Advances in Signal Processing, vol. 2007, Article ID 45789.

[9] G. J. Foschini et al., "Simplified processing for high spectral efficiency wireless communication employing multi-element arrays," IEEE Journal on Selected Areas in Communications, Nov. 1999.

[10] G. Ginis and J. M. Cioffi, "On the relation between V-BLAST and the GDFE," IEEE Communications Letters, Sept. 2001.

[11] S. Haykin, Adaptive Filter Theory, Prentice Hall, 3rd ed., 1996. 